\title{
Investigating Motivation Factors of Software Engineers in 6 Countries
}

\author{
Assad Saud Alzayed \\ Public Authority for Applied Education and Training \\ P.O.Box: 65130, Almansouriya, Kuwait 35652 \\ As.alzayed@paaet.edu.kw
}

\begin{abstract}
Much of the literature on motivation has noted the importance of motivation factors affecting project outcomes, particularly of software engineering teams working in the software development field. Although factors that motivate software engineering teams have been researched, little research has examined the motivation factors of software engineers within the GCC (Gulf Cooperation Council) countries. In order to examine the motivation factors that influence GCC software engineering teams, a survey questionnaire was distributed to software engineering practitioners in each of the six GCC countries. This study investigates motivation levels, the relationship between motivation levels and project outcomes, and the relationship between motivation factors and motivation levels. Results show that motivation levels vary across the region and that the relationship between motivation factors and motivation level vary from country to country. Not enough failed or partially successful projects were reported to make a claim about motivation level and project outcome, highlighting the difficulty of studying project outcomes. All 14 studied factors were significantly related to motivation level in the six countries combined, but there are individual countries where no factor is correlated with motivation level. This study's findings can assist software project managers working in the GCC countries or those dealing with global software development in the same region.
\end{abstract}

Keywords: Software development, Team motivation, Project outcome, Motivation factors.

\section{Introduction}

Motivation is considered to be the factor that has the biggest effect on practitioner productivity [1] and software quality management [2], and it is also important for staff retention [3], [4]. However, motivation is poorly understood and difficult to manage [5] and it is difficult to quantify [2]. It is also unclear what motivates software engineers in particular. Therefore, a model of motivation for software engineers is needed [5]. For example, software developers have been found to be motivated by the work that they do, including task identification, the variety of work, career planning, and developmental needs [6]. Understanding these factors and their relationship with motivation will help encourage better quality software, more sharing of skills, and an increase in productivity [6].

In previous research, software engineers in the US, Australia, Chile, and Vietnam were surveyed [7]. While it was found that the same six factors were significantly related to motivation levels in all 4 countries, there were differences between the countries as well. For example, in the US, software engineers were greatly influenced by practitioner factors, in Australia, motivation was influenced by project manager quality, in Chile, software engineers were concerned with practitioner and project management factors, and in Vietnam, software engineers were the only group motivated by external project factors. Thus, software engineers may respond to different factors within different countries. It is therefore important to understand the national culture when investigating motivational factors [8], as expectations for software developers can be quite different across cultures [9]. Differences in culture can influence team motivation [10].

Much research has been done on software engineers' motivations within Europe and the US. However, there is a lack of studies evaluating motivations of software engineers in the GCC countries. In this research, motivation levels and motivation factors of software engineers from six GGC countries are investigated and the factors are shown to be relevant only when looking at the six countries as a whole.

\section{Methodology}

This study aims to answer three questions: 1) What are the motivation levels within software engineering teams when working on projects in the GCC countries, and are these levels similar across countries? 2) What is the relationship between team motivation of software engineers and project outcomes in the GCC countries, and is the relationship similar across 
countries? 3) What factors contribute to software engineer motivation in the GCC countries, and are the factors the same across countries? This study used a survey to gauge motivation factors, such software development progress and project manager communication effectiveness, based on the perception of the software engineers who completed the survey. This section discusses the questionnaire design, selection of participants, and data analysis.

\subsection{Questionnaire Design}

This study utilized a questionnaire based on [7]. However, some questionnaire sections were not relevant to the current study. The original questionnaire was designed in 2000, after an extensive consultation with over ninety software developers and an extensive review of the literature on software development success and failure [11], which paved the way for a shorter version of the initial questionnaire. The questionnaire in this study focuses on key software development features: project management factors (e.g., project manager experience), software development factors (e.g., clearly defined project scope), external project factors (e.g., clients), and practitioner factors (e.g., rewards), and covered the professional team, management of the team, and the software development process (Table 1).

Table 1: Survey questions.

\begin{tabular}{|c|c|c|}
\hline Question Category & Scale (Abbreviated) & How variable was referenced \\
\hline \multicolumn{3}{|l|}{ Project Management (PM) Factors } \\
\hline Did the PM have full authority to carry out the project? & No authority - Full authority & Project manager authority \\
\hline Did the PM define vision driving the project? & No vision - Completed vision & Project manager vision \\
\hline How effective is PM in communicating with staff? & Poor - Good & Project manager communication \\
\hline How much experience did the PM have in managing projects? & Number of years work & $\begin{array}{l}\text { Project manager experience } \\
\text { (years) }\end{array}$ \\
\hline How do you rate the PM? & Poor - Good & Good project manager \\
\hline \multicolumn{3}{|l|}{ Software Development Factors } \\
\hline Did the project have a clearly defined scope? & Very poor - Partially - Very good & Scope well defined \\
\hline Did the project's scope change while the project was under way? & Extensive - Partial - No change & Scope changed \\
\hline Did the project utilize a defined development or other approach? & The name of the approach & Define development methodology \\
\hline Were any potential risk identified when the project began? & No risk - All risks identified & Potential Risks \\
\hline Did the project plan have any potential risks? & No risk - All risks incorporated & Risks incorporated \\
\hline Was risk assessment, control, and management carried out? & No risk - All risks controlled/managed & Risks reassessed \\
\hline \multicolumn{3}{|l|}{ External Project Factors } \\
\hline Were clients involved in the project? & No - Heavy client involvement & Customer involvement \\
\hline What level of confidence did the customer exhibit? & No - High confidence levels & Customer confidence \\
\hline Did the clients exhibit realistic expectations during the project? & Unrealistic - Realistic expectations & Customer expectation \\
\hline How can you rate the working environment of the project? & Poor - Good & Working environment \\
\hline Was project classified as a maintenance or development project? & Maintenance - Development & Project type \\
\hline \multicolumn{3}{|l|}{ Practitioner Factors } \\
\hline How were team members motivated in the project? & Low - High & Team Motivation \\
\hline Which areas of management was there (lack of) motivation? & All - No areas of management & Motivation/lack of motivation \\
\hline Did aggressive schedule affect motivation of the team? & Aggressive effect - No effect & Schedule effect on motivation \\
\hline Did project have enough staff to ensure that schedule is met? & Inadequate - Sufficient staffing & Adequate staff \\
\hline Did staff receive appreciation for working long hours? & No - Remarkable appreciation & Staff appreciation \\
\hline Did staff receive rewards for working long hours? & No - Good rewards & Staff rewards \\
\hline How can you rate the turnover rate during the project? & Low turnover - No turnover & Staff turnover \\
\hline Did key personnel stay throughout the project? & Many left - All employees stayed & Key personnel stayed \\
\hline How did staff members work together on the team? & Poor - Good & Team work \\
\hline What experience did you have as you worked on the project? & Bad - Good & Pleasant staff experience \\
\hline Do you consider the project to be a success? & $\begin{array}{l}\text { Successful - Partially successful - } \\
\text { Failure }\end{array}$ & Project outcome \\
\hline
\end{tabular}

Respondents answered the questions based on their recent experience in IT projects. A 4 or 5-point Likert scale was consolidated into a 2- or 3-point scale, depending on the question.

Project success cannot be defined in advance; respondents were allowed to provide their own definition of success. Success was ranked on a 3-point scale: successful, partially successful, and failure. Stakeholders perceive success differently [13]-[15] and practitioners will sometimes classify a project as successful when the organization classifies it as a partial 
failure [12]. As highlighted by [14], the success of a project correlates with engineer motivation. However, this study was unable to clearly define the connection between project outcome and motivation (see Section 3.2 for more discussion).

\subsection{Selection of Participants}

The participants were not randomly selected; instead, a convenient sample comprised of developers who were easily accessible was used. 163 respondents returned completed questionnaires, including 37 respondents from Bahrain, 36 respondents from Saudi Arabia (henceforth KSA), 12 respondents from Kuwait, 6 participants from Oman, and 36 respondents from the UAE. (As the sample size from Oman is so small, generalizations cannot be made about Oman.) There were 37 project managers, 20 customers, 28 users, 5 MIS managers, and 26 respondents in other management positions, and they were involved with software created for educational and financial institutions in the GCC countries. Participants were asked to answer all 25 questions (Table 1).

\subsection{Analysis}

An exploratory approach was adopted in analysis of the research findings. The data was investigated using contingency tables, bar charts, plots, and statistical tests. The data was incorporated into two-dimensional tables, which recorded each category of frequency. The contingency tables include data about GCC software engineers and information on project type, results, and team motivation levels. The analysis includes ordinal scale and categorical (yes/partially/no) data and suitable parametric and non-parametric statistical tests. The statistical tests help in analyzing the difference between project outcome and motivation factors among software developers in the GCC countries. Before these tests were carried out, team motivation was converted from a 5-point scale to a 3-point scale, as the data varied between 2 (low motivation) and 4 (high motivation). The participants did not use the most extreme values, such as very low or very high motivation levels.

For the first question (What are the motivation levels within software engineering teams when working on projects in the GCC countries, and are these levels similar across countries?), cross-tabulation was used to present team motivation levels for all projects. The Kruskal-Wallis was used test to find significant differences between team motivation levels within all six countries.

For the second question (What is the relationship between team motivation of software engineers and project outcomes in the GCC countries, and is the relationship similar across countries?), the chi square test of independence was necessary to carry out a comparison of the six countries. Respondents were placed into groups based on project success, and the MannWhitney U and Kruskal-Wallis tests were carried out to ascertain if motivation levels within the groups occur with the same distribution.

For the third question (What factors contribute to software engineer motivation in the GCC countries, and are the factors the same across countries?), the chi square test of independence was used to test if the factors in Table 1 can be considered to be motivational, which motivation factors are universal, and which factors are specific to a country. This requires the use of the Mann-Whitney U test, which compared medians of the provided responses for every factor examined to see if they differ among the respondents.

\section{Results}

This section first considers all projects in the dataset that are categorized as (1) development or maintenance and (2) successful, partially successful, or a failure. It then discusses the motivation for each country and overall in the GCC countries. Table 2 presents the general details of the data sample. The dataset includes combined data from all six countries. 127 projects were in software development (77\%) and only $36(22 \%)$ were in software maintenance.

Table 2: Number of respondents, and project type.

\begin{tabular}{|l|c|c|c|c|c|c|}
\hline Country & Projects & Respondents & \multicolumn{2}{|c|}{ Development } & \multicolumn{2}{c|}{ Maintenance } \\
\cline { 4 - 7 } & & & $\#$ & $\%$ & $\#$ & $\%$ \\
\hline Bahrain & 37 & 37 & 34 & $91 \%$ & 3 & $8 \%$ \\
\hline KSA & 36 & 36 & 26 & $72 \%$ & 10 & $27 \%$ \\
\hline Kuwait & 12 & 12 & 8 & $66 \%$ & 4 & $33 \%$ \\
\hline Oman & 6 & 6 & 5 & $83 \%$ & 1 & $17 \%$ \\
\hline Qatar & 36 & 36 & 31 & $86 \%$ & 5 & $14 \%$ \\
\hline
\end{tabular}




\begin{tabular}{|l|c|c|c|c|c|c|}
\hline UAE & 36 & 36 & 23 & $64 \%$ & 13 & $36 \%$ \\
\hline Total & 163 & 163 & 127 & $77 \%$ & 36 & $22 \%$ \\
\hline
\end{tabular}

\subsection{Motivation Levels}

In answering the first question (What are the motivation levels within software engineering teams when working on projects in the GCC countries, and are these levels similar across countries?), results show that motivation levels vary across the six countries (Table 3 and Fig. 1).

Table 3: Team motivation: overall and by country (\%).

\begin{tabular}{|l|c|c|c|c|c|c|c|}
\hline & Overall & UAE & KSA & Kuwait & Qatar & Bahrain & Oman \\
\hline Low motivation & 34.4 & 38.9 & 33.3 & 16.7 & 19.4 & 51.4 & 33.3 \\
\hline Average motivation & 34.4 & 30.6 & 50 & 33.3 & 30.6 & 27 & 33.3 \\
\hline High motivation & 31.3 & 30.6 & 16.7 & 50 & 50 & 21.6 & 33.3 \\
\hline Overall country motivation level & Low & Average & High & High & Low & Average \\
\hline
\end{tabular}

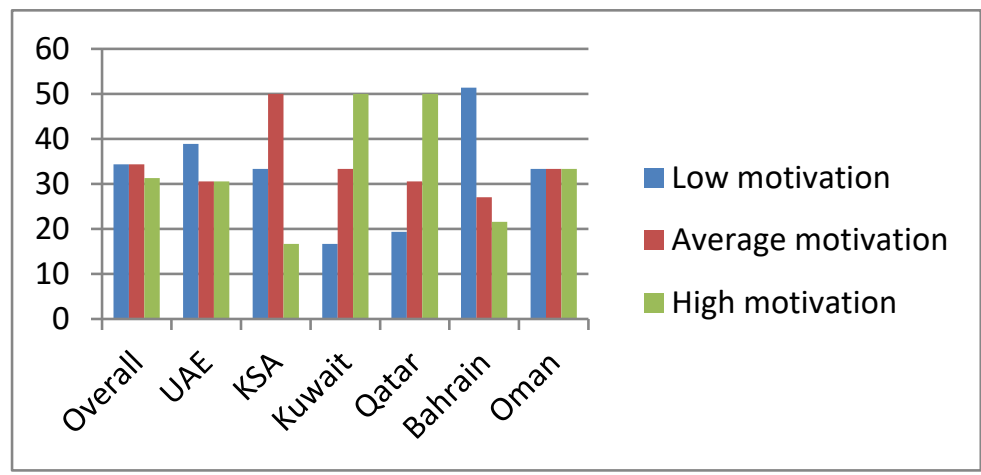

Fig. 1: Team motivation: overall and by country (\%).

KSA, and Oman had average team motivation medians, Kuwait and Qatar had high medians, UAE and Bahrain had a low median (Table 3). Overall, the motivation level for all six countries was average.

In order to show that motivation level is not the same across all countries, a Kruskal-Wallis test was applied. It shows that the null hypothesis (that motivation levels are the same across the GCC countries) is rejected. There are significant differences in team motivation levels across all countries (Kruskal-Wallis=13.694, p=.018). (Oman had very few respondents; thus we cannot rely on their results.) Kuwait and Qatar have the highest levels of motivation, and there is no significant difference between the two $(\mathrm{p}=.938)$. Bahrain has the lowest level of motivation, and the mean motivation level is significantly lower than Kuwait $(\mathrm{p}=.024)$ or Qatar $(\mathrm{p}=.002)$.

\subsection{Motivation Levels and Project Outcomes}

Of the projects discussed by the 163 respondents, 118 were considered to be successful (72\%), 45 were considered to be partially successful (27\%), and only 8 were considered to be failures $(5 \%)$ (Table 4$)$. 
Table 4: Number of successful, partially successful, \& failed projects.

\begin{tabular}{|l|c|c|c|c|c|c|c|}
\hline \multirow{2}{*}{ Country } & \multirow{2}{*}{ Total } & \multicolumn{2}{|c|}{ Successful } & \multicolumn{2}{c|}{ Partially successful } & \multicolumn{2}{c|}{ Failures } \\
\cline { 3 - 8 } & & $\#$ & $\%$ & $\#$ & $\%$ & $\#$ & $\%$ \\
\hline Bahrain & 37 & 32 & $86 \%$ & 5 & $14 \%$ & 0 & $0 \%$ \\
\hline KSA & 36 & 26 & $72 \%$ & 10 & $27 \%$ & 4 & $11 \%$ \\
\hline Kuwait & 12 & 4 & $33 \%$ & 8 & $66 \%$ & 0 & $0 \%$ \\
\hline Oman & 6 & 2 & $33 \%$ & 4 & $66 \%$ & 0 & $0 \%$ \\
\hline Qatar & 36 & 28 & $77 \%$ & 8 & $22 \%$ & 4 & $11 \%$ \\
\hline UAE & 36 & 26 & $72 \%$ & 10 & $27 \%$ & 0 & $0 \%$ \\
\hline Total & 163 & 118 & $72 \%$ & 45 & $27 \%$ & 8 & $5 \%$ \\
\hline
\end{tabular}

Those classified as failures included 4 from Qatar and 4 from KSA. Respondents considered most of their projects to be successful. Only respondents from Qatar and KSA reported failed projects. This rate seems far too low. Either the respondents' organizations had a high percentage of successful projects, or the respondents preferred to report only on successful projects. The respondents also might not have liked to consider any of their projects to be failures. Future research should investigate the reasons for the low reporting rate of unsuccessful projects from software engineers in the GCC countries.

Motivation level and project outcomes are listed in Table 5.

Table 5: Motivation level and project outcome by country.

\begin{tabular}{|l|l|c|c|c|c|c|c|}
\hline \multicolumn{2}{|c|}{} & UAE & KSA & Kuwait & Qatar & Bahrain & Oman \\
\hline \multirow{3}{*}{ Low motivation } & Success & 11 & 7 & 1 & 3 & 17 & 0 \\
\cline { 2 - 9 } & Partial success & 3 & 3 & 1 & 2 & 2 & 2 \\
\cline { 2 - 9 } & Failure & 0 & 2 & 0 & 2 & 0 & 0 \\
\hline \multirow{3}{*}{ Average motivation } & Success & 5 & 12 & 1 & 6 & 10 & 2 \\
\cline { 2 - 9 } & Partial success & 6 & 4 & 3 & 4 & 0 & 0 \\
\cline { 2 - 9 } & Failure & 0 & 2 & 0 & 1 & 0 & 0 \\
\hline \multirow{3}{*}{ Total motivation } & Success & 10 & 3 & 2 & 15 & 5 & 0 \\
\cline { 2 - 9 } & Partial success & 1 & 3 & 4 & 2 & 3 & 0 \\
\cline { 2 - 8 } & Failure & 0 & 0 & 0 & 1 & 0 & 2 \\
\hline \multicolumn{2}{|l|}{} & 36 & 36 & 12 & 36 & 37 & 6 \\
\hline
\end{tabular}

Additional testing was conducted on the successful projects $(\mathrm{N}=110),{ }^{1}$ as over two-thirds of the projects in the sample were considered to be successful. There were insufficient sample sizes at the country level to conduct significant testing for partial or unsuccessful projects. There are significant differences in team motivation levels across all countries (KruskalWallis=16.637, $\mathrm{p}=.005$ ) (Fig. 2). Qatar's team motivation level is significantly higher than the UAE's, KSA's, and Bahrain's $(\mathrm{p}=.034, .002, .000$, respectively). However, there is no significant difference in team motivation levels between the UAE and KSA. Despite the fact that all of Bahrain's projects were defined as successful, the software engineers' motivation is still low. This might be due to a lack of motivation factors, which is investigated in the next question.

\footnotetext{
${ }^{1}$ Oman was not included in the comparison test, due to its small sample size.
} 


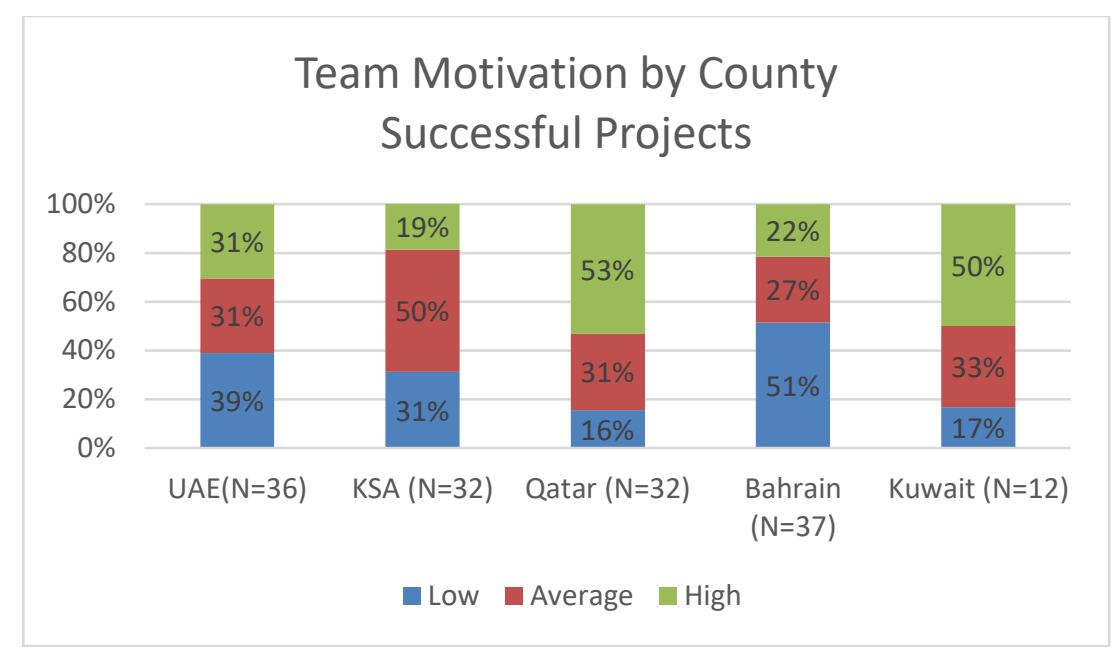

Fig. 2: Team motivation and successful project by country.

The second research question (What is the relationship between team motivation of software engineers and project outcomes in the GCC countries, and is the relationship similar across countries?) we investigated the relationship of team motivation with project outcome for all GGC countries. The null hypothesis is that there is no relationship between team motivation and project outcome. Using the Kruskal-Wallis, the null hypothesis is accepted, as there is no relationship between team motivation and project outcome (Kruskal-Wallis $U=.469 .5, \mathrm{p}=.220$ ) in the six countries. None of the projects in Kuwait, UAE and Bahrain were defined as completely failed. Therefore, we failed to see any positive or negative relationship between motivation and project outcome, or if the project is more likely to be successful when the team feels more motivated. However, Fig 2 and the plot graph in Fig 3 both show that software engineers in Qatar tend to be different from their counterparts in the other GCC countries; their software engineers are highly motivated when the project is successful, compared to other countries. When we collapsed the scale to three (Low, Average, High), Qatari team motivation data fails to show a relationship between motivation and project outcome - see the chi-square and Kruskal-Wallis tests (Fisher's Exact Test sig.=.151; Kruskal-Wallis sig.=0.094). We relate this result to the low number of failed projects in Qatar. We think that if there were more failed projects then we would have seen a relationship between the two variables.

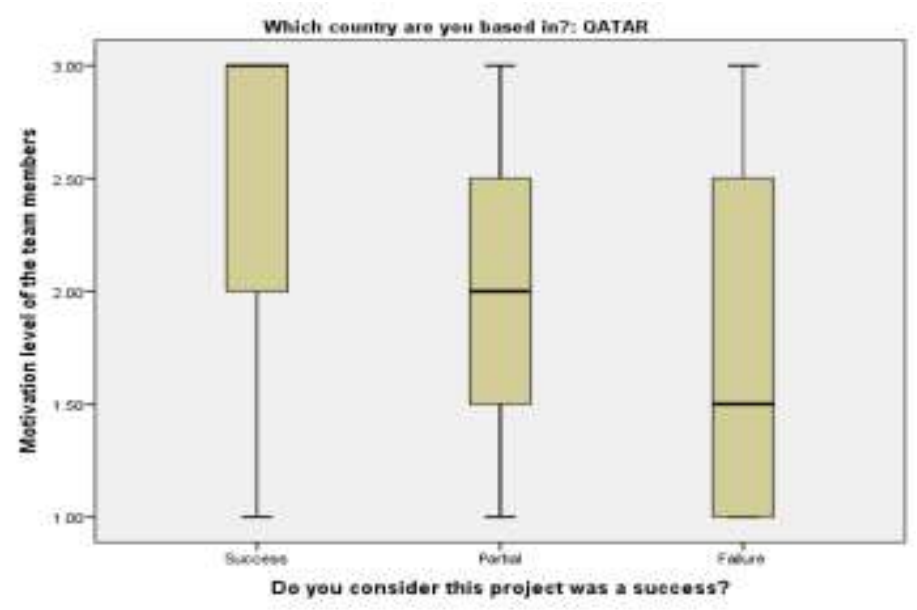

Fig. 3: Motivation level and project outcome for Qatar.

\subsection{Motivation Factors and Motivation Levels}

For the third question (What factors contribute to software engineer motivation in the GCC countries, and are the factors the same across countries?), motivation factors were investigated. There are four categories of factors that could influence 
motivation: project manager, software development, external, and practitioner factors. No factors are correlated with motivation in all six countries, some are not related at all, and some are only correlated within particular countries.

14 factors can be considered to be overall team motivation factors for at least some of GGC countries (Table 6).

Table 6: Motivation factors and their significance level with the GCC countries ( $\mathrm{p}<0.05$ in bold).

\begin{tabular}{|l|l|l|l|l|l|l|l|}
\hline & Overall & UAE & KSA & Kuwait & Qatar & Bahrain \\
\hline Project Manager (PM) Factors & $\mathbf{0 . 0 0 3}$ & 0.508 & 0.806 & 0.616 & $\mathbf{0 . 0 2 1}$ & $\mathbf{0 . 0 3 2}$ \\
\hline PM communication & $\mathbf{0 . 0 1 8}$ & 0.637 & 0.679 & 0.616 & 0.064 & 0.643 \\
\hline PM quality & \multicolumn{7}{|l|}{} \\
\hline Software Development Factors & $\mathbf{0 . 0 4 4}$ & 0.682 & 0.754 & 1.000 & 1.000 & 0.054 \\
\hline Was defined develop methodology used & $\mathbf{0 . 0 2 0}$ & 0.057 & 1.000 & 0.636 & 0.138 & 0.490 \\
\hline Risk identified at the start of project & $\mathbf{0}$ & \multicolumn{7}{|l|}{} \\
\hline Risk assessed and controlled, managed & $\mathbf{0 . 0 3 7}$ & 0.523 & 0.148 & 0.289 & 0.075 & 0.778 \\
\hline External Project Factors & $\mathbf{0 . 0 0}$ & 0.862 & 0.101 & 0.379 & $\mathbf{0 . 0 0 8}$ & $\mathbf{0 . 0 0 5}$ \\
\hline Level of confidence in project & $\mathbf{0 . 0 3 5}$ & 0.222 & 0.466 & 0.519 & 0.468 & 0.19 \\
\hline Customer expectation & $\mathbf{0 . 0 0 1}$ & 0.148 & 0.686 & 0.684 & $\mathbf{0 . 0 3 3}$ & 0.274 \\
\hline Working environment & $\mathbf{0 . 0 0 6}$ & 0.839 & 0.292 & 0.131 & 0.47 & $\mathbf{0 . 0 0 4}$ \\
\hline Practitioner Factors & $\mathbf{0 . 0 3 4}$ & 0.782 & 0.103 & 0.455 & 0.226 & 1.000 \\
\hline Project itself & $\mathbf{0 . 0 0 0}$ & 0.511 & 0.169 & 0.419 & 0.097 & 0.216 \\
\hline Technology used & $\mathbf{0 . 0 1 3}$ & 0.237 & 0.1000 & 0.209 & 0.718 & 0.059 \\
\hline Reward at the end of the project & $\mathbf{0 . 0 1 7}$ & 0.098 & 0.237 & 0.209 & 0.686 & 0.120 \\
\hline Adequate staff & $\mathbf{0 . 0 0 0}$ & 0.099 & 0.128 & 0.813 & $\mathbf{0 . 0 0 5}$ & 0.668 \\
\hline Key personnel &
\end{tabular}

Some factors are significantly associated with motivation in two countries but not others. Project manager communication and level of confidence are significantly related to motivation level in Qatar and Bahrain. Some are significantly associated with motivation in only one country: working environment and teamwork in Qatar and the project itself in Bahrain. None of the factors were significantly related to the UAE, KSA or Kuwait.

Overall, all of the factors were significantly related to motivation level of software engineers. Thus, these factors should be taken into consideration when working with software engineers in the GCC countries. However, to point out some of the key solutions for low motivation in the GCC countries:

- Good project manager: A good manager who can communicate and listen to every individual within his team, encourage work ownership, recognize team members when they have done a good work and recognize leader as will.

- Flexibility of work place and hours: This motivation element is a very important element as the software developer has the flexibility to work from different places within different hours. As the weather is hot in the GCC region and the software developers tend to work for long hours. It is recommended that the software developer can work from home or any place he can feel very comfortable with.

- Work environment: The work environment should be clean, quiet, and temperature is reasonable.

- Use of new technology: Project manager should always encourage his team to use new technology and send them for training if they wanted to.

- Level of confidence in project: The software engineers should have a high confidence in the project that they are working on so that they can have commitment and loyalty to the project.

\subsection{Comparison to Previous Research}

Comparing these results to [7], Qatar is similar to Vietnam; Vietnam has a much higher percentage of highly motivated team members when the project is successful, compared to other countries, such as Australia, the US, and Chile. Qatar also has a much higher level of motivation than other countries (although the relationship of motivation to project outcome cannot 
be tested). On the other hand, this GCC sample produces different results than [7], where it was found that software engineering teams in four different countries (the US, Australia, Chile, and Vietnam) were motivated by the same six factors: project management skills, risks assessed and controlled, customer confidence, good working environment, teamwork, and pleasant work experience. In three out of the six GCC countries, none of the factors were correlated with motivation, one country did not have enough data to run statistics on, and two out of the six shared two factors: project manager communication and level of confidence. Other factors were correlated to motivation in one country only: working environment and teamwork in Qatar and the project itself in Bahrain.

\subsection{Validity}

The questionnaire used in this research has been used successfully many times with other software developers from different countries [11], [14]. Therefore, the questionnaire is a valid instrument for exploring motivational factors for software engineers, and using the same questionnaire allows us to compare results with previous studies.

Because our research sample is a convenient sample, not a random sample, the results are not as reliable as a random survey would be, as convenient samples might be biased. As this research was conducted in the GCC countries only, which is a small part of the world, the findings cannot be generalized, and are limited to the sample population at the time the survey was conducted.

Ordinal data and a non-parametric statistics were used for the analysis. This study is reliable since the variables are taken from experienced software engineers who have knowledge of different software development projects. Limitations of the study include the small number of projects in Kuwait and particularly Oman and the low number of failed projects overall.

\section{Conclusion}

The results of this study show that motivation levels of software engineers vary across the six GCC countries. The UAE, KSA, and Oman had average team motivation medians, Kuwait and Qatar had high medians, and Bahrain had a low median. Project outcomes could not be related to motivation as very few projects were labeled as failures and not enough were labeled partially successful. Finally, project manager communication and level of confidence are significantly related to motivation level in both Qatar and Bahrain, working environment and teamwork is significantly related to motivation level in Qatar, and the project itself is significantly related to motivation level in Bahrain. All factors listed in Table 6 were significantly related to motivation level when looking at the data from the GCC countries as a whole.

This contradicts the findings in [7], where only six factors were found to be significantly related to motivation levels of software engineers in USA, Australia, Vietnam, and Chile. All of the factors studied in this research (Table 6) are significantly related to motivation level when looking at the data from the GCC countries as a whole, but not when looking at the data specifically within each country. The research results show that more research needs to done to understand motivation levels of software engineering practitioners working in GCC countries. They also show that the project manager needs full authority to manage the project, to be highly experienced, and to know how to deal with multinational teams.

Further research should be conducted to investigate a similar sample, with more data collected on failed projects, in order to measure correlations between motivation and project outcome. The research results would be valuable for project managers working in the GGC countries and also for global software projects taking place in the same area.

\section{Acknowledgements}

I would like to thank the Public Authority of Applied Education and Training (PAAET) and the manager of the research department for their full support and understanding during this research and for providing the funds needed to complete this work. The research paper No. BS-17-10.

\section{References}

[1] B. W. Boehm, Software Engineering Economics. Englewood Cliffs, NJ: Prentice-Hall, 1981.

[2] S. McConnell, "Avoiding classic mistakes [software engineering]," IEEE Software, vol. 13, no. 5, pp. 111-112, 1996.

[3] R. Agarwal and T. Ferrat, "Enduring practices for managing IT professionals," Communications of the ACM, vol. 45, no. 9, pp. 73-79, 2002.

[4] P. C. Lee, "The social context of turnover among information technology professional," in Proceedings of the 2002 ACM SIGCPR Conference on Computer Personnel Research, Kristiansand, Norway, 2002, pp. 145-153. 
[5] H. Sharp, N. Baddoo, S. Beecham, T. Hall, and H. Robinson, "Models of motivation in software engineering," Information and Software Technology, vol. 51, no. 1, pp. 219-233, 2009.

[6] S. Beecham, N. Baddoo, T. Hall and H. Sharp, "Motivation in software engineering: A systematic literature review," Information \& Software Technology, vol. 50, nos. 9-10, pp. 860-878, 2008.

[7] J. M. Verner, M. A. Barbar, N. Cerpa, S. Beechman and T. Hall, "Factors that motivate software engineering teams: A four country empirical study," J. of Software and Systems, vol. 92, pp. 115-127, 2014.

[8] A. Cater-Steel and M. Toleman, "The impact of national culture on software engineering practices," Int. J. of Technology, Policy \& Management, vol. 8, no.1, pp. 76-90, 2008.

[9] J. M. Burn, L. C. Ma and E. M. Ng Tye, "Managing IT professionals in a global environment," SIGCPR Comput. Pers, vol. 16, no. 3, pp. 11-19, 1995.

[10] J. M. Verner, S. Beecham and N. Cerpa, "Stakeholder dissonance: Disagreements on project outcome and its impact on team motivation across three countries," ACM SIGMIS, Vancouver, BC, 2010, pp. 25-33.

[11] J. M. Verner and W. Evanco, "In-house software development: What software project management practices lead to success?" IEEE Software, vol. 22, no. 1, 86-93, 2005.

[12] U. M. Al-Turki, "An exploratory study of ERP implementation in Saudi Arabia," Production Planning \& Control, vol. 22 no. 4, 403-413, 2001.

[13] M. Shokri-Ghasabeh and K. Kavoousi-Chabok, "Generic project success and project management success criteria and factors: Literature review and survey," WSEAS Transactions on Bus. \& Economics, vol. 6, no. 8, 2009, pp. 456-468.

[14] J. Pereira, N. Cerpa, J. Verner, M. Rivas and J. D. Procaccino, "What do software practitioners really think about project success: A cross-cultural comparison," J. of Systems \& Software, vol. 81, no. 6, 897-907, 2008.

[15] K. R. Linberg, "Software developer perceptions about software project failure: A study," J. of Systems \& Software, vol. 49, no. 2/3, pp. 177-192, 1999. 